\title{
Sortir de l'obscurité
}

\author{
En psychiatrie aussi, l'indemnisation sera à l'avenir liée à la prestation. A la différence \\ de ce qui prévaut en somatique aiguë, il n'y aura pas (pas encore), depuis 2012, de \\ forfait par cas lié au diagnostic. Mais d'ici 2015 un système de rémunération for- \\ faitaire uniforme au niveau national devra être introduit pour la psychiatrie sta- \\ tionnaire. Directeurs, médecins chefs, et directeurs des soins se sont réunis début \\ avril 2011 pour discuter ensemble de la situation.
}

Irène Dietschi

\section{Correspondance:}

Dr Graziella Giacometti Bickel Présidente Association suisse des médecins-cheffes et médecins-chefs en psychiatrie ASMP

Postgasse 17

CH-3000 Berne 8

Tél. 0313138849

welcome@

swissmentalhealthcare.ch
Pour la psychiatrie, que signifie en somme «orientation sur la prestation» et que faut-il entendre par multi-professionnalisme? C'est à cette question qu'a été consacré, sur invitation de la Clinique psychiatrique Königsfelden, à Brugg, le premier symposium de Swiss Mental Healthcare (SMHC), association des cliniques et services psychiatriques de Suisse (voir encadré). Sujet sensible, car le débat ne portait pas seulement sur la manière de calculer adéquatement les prestations en psychiatrie, mais aussi sur les effets que peut avoir un nouveau modèle de tarif sur la collaboration entre groupes professionnels impliqués: $\mathrm{Si} \mathrm{l'on}$ travaille selon le système de la prestation, cela changet-il à la base la perception du rôle du médecin, du soignant et du thérapeute dans les directions des cliniques? La relation avec les patients en est-elle modifiée?

«Il faut que chacun fasse ce qu'il peut faire le mieux, l'important devant être la transparence», a déclaré d'emblée Ursula Fringer, présidente de SMHC et présidente des directeurs de cliniques; elle voit nettement des chances dans un financement orienté sur la prestation. Il est primordial toutefois que les futurs mandats de prestation soient définis non par les politiques seuls, mais en concertation avec la psychiatrie institutionnelle. «Il faut expliquer et montrer comment fonctionne une institution", ajoute Harald Müller, qui préside la Conférence des directrices et directeurs des prestations de soins des institutions psychiatriques de Suisse (KPP). «Pour les assurances, par exemple, la psychiatrie est une boîte noire. Elles y versent de l'argent mais ignorent ce qui en résulte.»

\section{SwissMentalHealthcare}

Swiss Mental Healthcare est l'association des directrices et directeurs, des médecins-chefs ainsi que des directrices et directeurs des soins des cliniques et hôpitaux psychiatriques de Suisse. Elle entend occuper un rôle d'interlocuteur entre la psychiatrie institutionnelle et tous les acteurs travaillant dans le domaine de la santé. Cette alliance, nouvelle en Suisse, veut s'engager pour la psychiatrie institutionnelle dans le domaine stationnaire comme dans le secteur ambulatoire ainsi que dans les cliniques de jour. Swiss Mental Healthcare soutient, par des informations ciblées et des faits documentés, un financement des soins psychiatriques équitable, orienté sur la prestation.

\section{Forfaits journaliers liés à la prestation}

Un financement plus transparent est donc demandé. Mais comment procéder? En psychiatrie, il n'est guère possible de pronostiquer les coûts sur la base du diagnostic. Sur ce point, les intervenant-e-s à la session de Königsfeld étaient unanimes. En effet: contrairement à la situation en somatique aiguë, on ne peut pas prédire le processus de guérison chez les malades psychiques. «Pas plus que le risque de rechutes il n'est possible d'évaluer le cours de la maladie dans son ensemble», a déclaré la Dresse Graziella Giacometti Bickel, présidente de l'Association des médecins-chefs (ASMP).

Il existe une autre formule de forfait par cas: le forfait journalier. En Allemagne, on met au point actuellement un système de financement dont l'élément central consiste en de tels forfaits journaliers pour le traitement stationnaire et partiellement stationnaire, et cela indépendamment des prestations effectivement fournies.

Le système devrait être une incitation à engager des prestations de manière efficace et appropriée («pay for performance»). L'introduction est prévue pour 2013.

Pour le moment, un vaste processus de calculation est en cours, avec description des prestations et de l'utilisation de leurs ressources. Au symposium de SMHC, Georg Oppermann, directeur des prestations de soins à l'hôpital de district d'Augsburg et membre du Deutscher Pflegerat, a donné un aperçu du déroulement de la journée en clinique. Les prestations fournies directement (thérapie et diagnostic) sont toujours recensées en unités de 25 minutes, ni plus ni moins. Les coûts restants (y compris la «simple» présence) sont calculés dans un «modèle de pondération». Distinction est faite entre traitement intensif et ordinaire et entre diagnostics importants et secondaires. Du fait qu'une rétribution forfaitaire est perçue pour chaque journée de traitement, il en découle pour le patient individuellement des forfaits journaliers qui changent chaque jour. Le système, qui est à l'essai, est encore peu satisfaisant pour ses utilisateurs, comme l'indique Georg Oppermann: «La charge administrative est énorme.» Son hôpital a engagé des documentalistes spécialement chargés du relevé des prestations. Mais 
le système ne reconnaît que ce qui a été saisi et encodé dans le cadre de la classification définie. «Il s'ensuit que tous les participants veulent coder le plus grand nombre possible d'unités de thérapie, voire parfois en créer de nouvelles. Ils s'adaptent aux intervalles de 25 minutes.» On ne peut éviter un «upcoding». Pourtant, plus de codage ne signifie au total pas plus d'argent pour la psychiatrie.

Oppermann reconnaît néanmoins au système certains aspects positifs: Il a renforcé la cohésion entre médecins, soignants et autres groupes professionnels. L'intérêt commun fait qu'on tire à la même corde.

\section{Mêmes normes au CHUV}

Une documentation précise des prestations fournies peut certainement contribuer à la qualité. C'est ce qu'a démontré dans son exposé Patrice Guex, chef de la division de psychiatrie générale et du département de psychiatrie de l'Hôpital universitaire vaudois (CHUV). $\mathrm{Au} \mathrm{CHUV}$, la psychiatrie est évaluée exactement selon les mêmes critères économiques que les autres départements. Le monitoring des prestations psychiatriques et le management de la qualité (gouvernance clinique) vont de soi car «il importe de très bien motiver ce que l'on fait pour justifier son budget», explique Patrice Guex.

Son département travaille notamment avec le système HoNOS, originaire du Royaume-Uni; à l'aide de cet instrument de mesure, sur une échelle de 1 à 12 , on définit le degré de gravité d'un problème psychiatrique, et par là les besoins du patient. Les collaborateurs n'ont aucune réticence à entreprendre ces évaluations, au contraire: «Du fait qu'ils documentent leurs prestations, ils prennent mieux conscience de leur activité» commente Patrice Guex, «et c'est finalement au bénéfice du patient, qui est au centre.»

Autre mot vedette à la session SMHC de Brugg: le «multiprofessionnalisme». Les organisateurs sont partis de l'idée qu'un nouveau système de financement pouvait être une chance de briser des structures dépassées et de trouver de nouvelles formes de collaboration. «Dans les cliniques, qui fait quoi, comment, et qui le fait le mieux? Ce sont des questions que nous voulons élucider». Tel a été l'appel de la présidente Ursula Fringer.

\section{Superflue, la «bénédiction du médecin»?}

L'entretien entre Samuel Rom, psychologue spécialiste en psychothérapie FSP, et le psychiatre Erich Seifritz, directeur de clinique au PUK Zurich, a montré la divergence des réponses. Samuel Rom, qui dirige en qualité de CEO la clinique Schützen à Rheinfelden, a fait valoir que la focalisation sur la médecine dans les cliniques - et par là la domination des psychiatres - n'est plus de mise. Il s'appuie en particulier sur son statut professionnel: «Dans les cliniques, à part 500 médecins, quelque 350 psychologues sont en activité. Nous sommes bien formés, nous effectuons des examens et des thérapies. Dans notre profession, dès le premier jour de longues années de formation, le patient occupe la place centrale. Bref, nous fournissons un très bon travail.» La nouvelle loi sur les professions de la psychologie reconnaît au psychologue la capacité de résoudre des problèmes médicaux. Il n'est pas acceptable que les psychologues doivent faire les frais de la pénurie de psychiatres. «Si l'on recourt à notre expertise, alors nous réclamons aussi les compétences qui vont de pair.» La «bénédiction suprême» du médecin n'a plus sa place dans une équipe pluridisciplinaire.

Réplique d'Erich Seifritz: «Samuel Rom sous-estime la partie médicale de la formation en psychiatrie. Notre compréhension de la maladie se fonde sur le modèle bio-psycho-social. Dans ce tableau, la psychothérapie est certes une partie importante du traitement mais elle ne couvre pas les aspects biologiques, ni même, dans une certaine mesure, les aspects sociaux.» $\mathrm{Au}$ PUK, où le travail est fortement interdisciplinaire, dans l'unité de psychothérapie récemment créée, les psychologues ont souvent la gestion d'un cas - à condition que la partie médicale soit assurée là où le besoin existe. Une voie possible pour que les psychologues soient investis des compétences médicales consisterait en des études «postgrade» sur les contenus médicaux de rigueur en psychiatrie, selon le modèle hollandais. Il existe d'autres constructions dans le domaine des soins, mais avec d'autres interfaces vers la médecine. «Je n'ai rien contre les équipes interdisciplinaires» conclut Seifritz, «mais je m'oppose au nivellement.»

\section{Valorisation des soins}

Quoi qu'il advienne de ce débat, la prise en charge du patient en psychiatrie changera à l'avenir. Cela tient entre autres à une augmentation des nombres de cas due à des facteurs démographiques - en regard d'un manque de soignants et d'une pénurie encore plus marquée de médecins. C'est la réflexion livrée par Dirk Richter, de la Haute Ecole de santé du canton de Berne, dans son exposé sur les perspectives en matière de soins. Il est à prévoir que l'on passera à une prise en charge intégrée, majoritairement ambulatoire. Plus qu'aujourd'hui, l'assistance au patient psychiatrique sera surtout de l'aider à conserver son statut dans la société et son réseau social («ré-insertion»). Les mesures esquissées par Dirk Richter portent sur l'«empowerment» du patient. Il s'agit de le rendre capable de se considérer comme le centre moteur de sa vie, par exemple au moyen de coaching ou d'une méthode appelée «motivational interviewing» qui vise à se rendre soi-même capable par le développement de sa motivation. Les soignants sont parfaitement équipés pour de telles tâches, a affirmé l'orateur. Une étude conduite auprès de sa Haute Ecole spécialisée a révélé qu'aux yeux des soignants, c'est dans l'orientation du patient que résident essentiellement la qualité et l'utilité de leur travail. «La psychiatrie de l'avenir devra changer d'orientation dans de nombreux domaines» résume Richter. Le secteur des soins pourra assumer et redéfinir pour lui-même une partie des tâches nécessaires à cette évolution et qui jusqu'ici n'ont pas été suffisamment prises en compte. La profession de soignant en sera valorisée. 